\title{
On Isomorphic Factorizations of Circulant Graphs
}

\author{
Brian Alspach* and Danny Dyer \\ Department of Mathematics and Statistics \\ University of Regina \\ Regina, Saskatchewan \\ Canada S42 0A2 \\ Donald L. Kreher \\ Department of Mathematical Sciences \\ Michigan Technological University \\ Houghton, Michigan \\ U. S. A. 49931
}

September 2, 2005

\begin{abstract}
We investigate the conjecture that every circulant graph $X$ admits a $k$-isofactorization for every $k$ dividing $|E(X)|$. We obtain partial results with an emphasis on small values of $k$.

keywords: circulant graph; isomorphic factorization; $k$-isofactorization

\section{Introduction}

Given a graph $X$, we call a partition of the edge set $E(X)$ into parts $E_{1}, E_{2}, \ldots, E_{k}$ an isomorphic factorization provided that the subgraphs $\left\langle E_{1}\right\rangle,\left\langle E_{2}\right\rangle, \ldots,\left\langle E_{k}\right\rangle$ induced by the respective edge sets are mutually isomorphic. The first paper on graph decompositions, with isomorphic factorizations as the viewpoint [7], appeared in 1978. It led to many papers on the topic and interest in the problem continues to the present.

In order to simplify terminology, henceforth we shall refer to an isomorphic factorization of a graph into subgraphs with $k$ edges as a $k$-isofactorization. Many of the early papers on isofactorizations dealt with graphs possessing considerable symmetry. The corresponding results tended to follow the maxim that if $k$ divides the number of edges in the graph, then there is a $k$-isofactorization
\end{abstract}

\footnotetext{
${ }^{*}$ This research supported by NSERC under Grant A-4792
} 
of the graph. This led the first author to pose the following three problems [1]. If $k$ divides the number of edges in $X$, then does $X$ have a $k$-isofactorization whenever $X$ is in the family of circulant graphs, or the family of Cayley graphs, or the family of vertex-transitive graphs?

We consider the $k$-isofactorization problem for the family of circulant graphs in this paper. The circulant graph $X=\operatorname{circ}(n ; S)$ is the graph with vertex set $\left\{u_{0}, u_{1}, \ldots, u_{n-1}\right\}$, and an edge joining vertices $u_{i}$ and $u_{j}$ if and only if $j-i \in S$, where $S \subseteq \mathbb{Z}_{n} \backslash\{0\}$ and $s \in S$ if and only if $-s \in S$. We denote an edge joining $u_{i}$ and $u_{j}$ by $u_{i} u_{j}$. The set $S$ is called the connection set of $\operatorname{circ}(n ; S)$. For example, $\operatorname{circ}(8 ;\{1,2,4,6,7\})$ is given in Figure 1 . The edge joining $u_{i}$ and $u_{j}$ is said to have length equal to the residue in the range $1,2, \ldots,\lfloor n / 2\rfloor$ that is congruent to $j-i$ or $i-j$ modulo $n$.

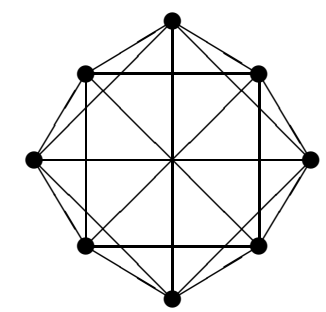

The circulant graph $\operatorname{circ}(8 ;\{1,2,4,6,7\})$.

The general problem for circulant graphs is to show that $\operatorname{circ}(n ; S)$ has a $k$ isofactorization whenever $k$ divides $(n|S| / 2)$, the number of edges in the graph. The number of subgraphs must be $(n|S| / 2 k)$. There has been a little work done on this problem. In [10] it is shown that $\operatorname{circ}(n ; S)$ has a $k$-isofactorization whenever $|S|$ is a prime and $k$ divides $n|S| / 2$. In [11] it is $\operatorname{shown}$ that $\operatorname{circ}(n ; S)$ has a $k$-isofactorization whenever $|S|=4$ and $k$ divides $2 n$.

\section{Some Constructions}

In this section we present some special constructions that produce isofactorizations. These constructions are used to handle many cases. The first contruction involves matchings.

2.1 Definition. A $k$-matching in a graph $X$ is a set of $k$ independent edges, that is, $k$ edges that have no common vertices.

2.2 Lemma. Let $X$ be a regular graph of order $n$ and valency 1 or 2 . If $k$ is a proper divisor of $|E(X)|$, then $X$ can be decomposed into $k$-matchings except when $n=2 k$ and $X$ has an odd order component.

Proof. If $X$ is regular of valency 1 , then $X$ itself is an $\frac{n}{2}$-matching. If $k$ is a proper divisor of $|E(X)|$, then $k \mid n / 2$. We simply partition the $\frac{n}{2}$-matching into $k$-matchings. 
We move to the valency 2 case. Let

$$
C_{1}, C_{2}, \ldots, C_{t}
$$

be the $t$ cycles comprising the components of $X$. Let the respective orders be $\ell_{1}, \ell_{2}, \ldots, \ell_{t}$. We know that

$$
\sum_{i=1}^{t} \ell_{i}=n
$$

and that $k$ is a proper divisor of $n$. Thus, we want to find $n / k k$-matchings that partition $E(X)$. The proof of Theorem 1.1 in [6] gives us the result for all $n / k \geqq 3$. When $n / k=2$, we need a proper edge coloring of $X$ with 2 colors. This is possible exactly when every cycle has even length. This completes the proof of the theorem.

2.3 Theorem. If $X=\operatorname{circ}(n ; S)$ is connected, $k$ is a proper divisor of $n$, and $k$ divides $|E(X)|$, then there is a $k$-isofactorization of $\operatorname{circ}(n ; S)$ into $k$-matchings.

Proof. If $n$ is even, consider the special divisor $k=n / 2$ first. By the main result in [9], $\operatorname{circ}(n ; S)$ has a 1 -factorization. Each 1 -factor is a $k$-matching and we are done in this case.

We may assume that the divisor $k$ satisfies $k<n / 2$. We write $\operatorname{circ}(n ; S)$ as the union of circulant subgraphs $\operatorname{circ}(n ;\{ \pm s\}), s \in S$ and use Lemma 2.2 to independently decompose each into $k$-matchings. Note that this also works for $s=n / 2$ because if $k$ divides $n$ and $|E(X)|$, then $k$ also divides $n / 2$ when $n / 2 \in S$. We may apply Lemma 2.2 here as well.

Let $\rho$ be the permutation $\left(\begin{array}{llllll}u_{0} & u_{1} & u_{2} & \cdots & u_{n-1}\end{array}\right)$. The permutation $\rho$ is an automorphism of every circulant graph. It also affords us a convenient way to describe subgraphs of a circulant graph. If $Y$ is a subgraph of $\operatorname{circ}(n ; S)$ and $V(Y)=\left\{u_{i_{1}}, u_{i_{2}}, \ldots, u_{i_{t}}\right\}$, then $\rho(Y)$ is the subgraph of $\operatorname{circ}(n ; S)$ with vertex set $\left\{\rho\left(u_{i_{1}}\right), \rho\left(u_{i_{2}}\right), \ldots, \rho\left(u_{i_{t}}\right)\right\}$ and has an edge joining $\rho\left(u_{i_{\alpha}}\right)$ and $\rho\left(u_{i_{\beta}}\right)$ if and only if there is an edge joining $u_{i_{\alpha}}$ and $u_{i_{\beta}}$. We define $\rho^{j}(Y)=\rho\left(\rho^{j-1}(Y)\right)$.

2.4 Definition. Given $n$ vertices cyclically labelled $u_{0}, u_{1}, \ldots, u_{n-1}$, let $\ell_{1}, \ell_{2}, \ldots, \ell_{t}$ be a sequence of integers satisfying $1 \leqq \ell_{1}<\ell_{2}<\cdots<\ell_{t} \leqq n / 2$. A zig-zag path using the lengths $\ell_{1}, \ell_{2}, \ldots, \ell_{t}$ is a path of the following form, where all subscript computations are performed in $\mathbb{Z}_{n}$. Its initial vertex is some $u_{i}$. The first edge goes from $u_{i}$ to $u_{i+\ell_{1}}$. The next edge goes from $u_{i+\ell_{1}}$ to $u_{i+\ell_{1}-\ell_{2}}$. We then use an edge of length $\ell_{3}$ by going from $u_{i+\ell_{1}-\ell_{2}}$ to $u_{i+\ell_{1}-\ell_{2}+\ell_{3}}$. We continue in this way going back and forth with edges of increasing length from our list of edge lengths until all lengths are used.

2.5 Theorem. If $k$ divides $|S| / 2$, then the $g r a p h \operatorname{circ}(n ; S)$ has a $k$-isofactorization into zig-zag paths of length $k$.

Proof. The hypothesis that $k$ divides $|S| / 2$ implies that $|S| / 2$ is an integer, so that either $n$ is odd or $n$ is even and $n / 2 \notin S$. Choose $t=\frac{|S|}{2}$ distinct 
lengths from $S$ so that $\ell_{1}<\ell_{2}<\cdots<\ell_{t}<\frac{n}{2}$. Since $k$ divides $t=|S| / 2$, we may partition $\left\{\ell_{1}, \ell_{2}, \ldots, \ell_{t}\right\}$ into $t / k$ parts $\mathcal{P}_{1}, \mathcal{P}_{2}, \ldots, \mathcal{P}_{t / k}$ of cardinality $k$. For each part $\mathcal{P}_{i}$, we take a zig-zag path $P_{i}$ using the lengths of $\mathcal{P}_{i}$. It is clear that the paths $P_{1}, P_{2}, \ldots, P_{t / k}$ under the action of $\rho, \rho^{2}, \ldots, \rho^{n-1}$ partition the edges of $\operatorname{circ}(n ; S)$ as required.

We remind the reader that the size of a graph is the number of edges in the graph.

2.6 Theorem. If $k$ divides $|S| / 2$, then the $g r a p h \operatorname{circ}(n ; S)$ has a $k$-isofactorization into stars of size $k$.

Proof. Again, the hypothesis that $k$ divides $|S| / 2$ implies that either $n$ is odd or $n$ is even and $n / 2 \notin S$. We follow the same procedure as used for Theorem 2.5 to obtain the partition $\mathcal{P}_{1}, \mathcal{P}_{2}, \ldots, \mathcal{P}_{t / k}$. However, now we form stars of size $k$, rather than zig-zag paths, and use the same rotations as above.

We have been emphasizing the size of the isofactors, but it is natural to wonder if divisors of the order of the circulant also lead to easy isofactorizations in terms of the number of the factors. The next result shows that this is the case.

2.7 Theorem. If $d \mid n$, then $\operatorname{circ}(n ; S)$ has an $\frac{n|S|}{2 d}$-isofactorization except for the case that $n$ is even, $n / 2 \in S$, and $n / d$ is odd.

Proof. If $n$ is odd or $n$ is even and $n / 2 \notin S$, the elements $s$ and $-s$ are distinct. Hence, in these cases, we may partition the connection set into two parts $A$ and $-A$, where for each $\pm s \in S$, we arbitrarily put one of them in $A$ and the negative in $-A$.

Choose $V_{0}=\left\{u_{i}: i \equiv 0(\bmod d)\right\}$. Now let $E_{0}=\left\{u_{i} u_{i+a}: u_{i} \in V_{0}, a \in A\right\}$. Let $X_{0}$ be the subgraph of $\operatorname{circ}(n ; S)$ induced by $E_{0}$. It is then clear that $X_{0}, \rho\left(X_{0}\right), \rho^{2}\left(X_{0}\right), \ldots, \rho^{d-1}\left(X_{0}\right)$ are $d$ mutually isomorphic subgraphs partitioning $E(\operatorname{circ}(n ; S))$ into subgraphs of size $n|S| / 2 d$.

Alternately, we may take $V_{0}^{\prime}=\left\{u_{0}, u_{1}, \ldots, u_{n / d-1}\right\}$. Again let $E_{0}^{\prime}=\left\{u_{i} u_{i+a}\right.$ : $\left.u_{i} \in V_{0}, a \in A\right\}$, and $X_{0}^{\prime}$ be the subgraph of $\operatorname{circ}(n ; S)$ induced by $E_{0}^{\prime}$. Then the subgraphs $X_{0}^{\prime}, \rho^{n / d}\left(X_{0}^{\prime}\right), \rho^{2 n / d}\left(X_{0}^{\prime}\right), \ldots, \rho^{(d-1) n / d}\left(X_{0}^{\prime}\right)$ are $d$ mutually isomorphic subgraphs partitioning $E(\operatorname{circ}(n ; S))$ into subgraphs of size $n|S| / 2 d$.

In the case that $n$ is even and $n / 2 \in S$, we may assume that $n / d$ is even by hypothesis. Then both $u_{i}$ and $u_{i+n / 2}$ belong to $V_{0}$ so that $E_{0}$ contains the edges of length $n / 2$ joining them. Thus, again $X_{0}, \rho\left(X_{0}\right), \rho^{2}\left(X_{0}\right), \ldots, \rho^{d-1}\left(X_{0}\right)$ are $d$ mutually isomorphic subgraphs partitioning $E(\operatorname{circ}(n ; S))$ into subgraphs of size $n|S| / 2 d$.

We now present a theorem which unifies and expands the results of this section.

2.8 Theorem. Let $X=\operatorname{circ}(n ; S)$ be a connected circulant graph of order $n$. If $k$ divides $|E(X)|$ and either $k$ properly divides $n$ or $k$ divides $|S|$, then there is a $k$-isofactorization of $X$. 
Proof. If $k$ is a proper divisor of $n$, then Theorem 2.3 gives us the desired conclusion for this subcase. If $k \mid \frac{|S|}{2}$, then the conclusion follows from either Theorem 2.5 or Theorem 2.6 when $n$ is odd.

If $k \mid \frac{|S|}{2}$ and $n$ is even, then the length $n / 2 \notin S$ because $n / 2=-n / 2$ and $|S|$ is even. So $S$ has the form $\left\{ \pm \ell_{1}, \pm \ell_{2}, \ldots, \pm \ell_{t}\right\}$. We now use the proofs of either Theorem 2.5 or Theorem 2.6 to obtain isofactorizations into either paths of length $k$ or stars of size $k$.

If $k=|S|$, then $n$ must be even because $k$ divides $|E(X)|=\frac{n|S|}{2}$. Suppose $S$ contains the lengths $\pm \ell_{1}, \pm \ell_{2}, \ldots, \pm \ell_{t}$ and possibly the diameter length $n / 2$. Start a zig-zag path $P_{1}$ from vertex $u_{0}$ using all lengths except $n / 2$. Start a zig-zag path $P_{2}$ from $u_{n / 2}$ using all lengths except $n / 2$. If $n / 2$ is not in $S$, $\left|P_{1} \cup P_{2}\right|=2 t=|S|=k$ and, thus, $P_{1} \cup P_{2}, \rho\left(P_{1} \cup P_{2}\right), \ldots, \rho^{n / 2-1}\left(P_{1} \cup P_{2}\right)$ is a $k$-isofactorization of $X$. On the other hand, if $n / 2 \in S$, form a single path $P$ by adding the edge from $u_{0}$ to $u_{n / 2}$ to $P_{1} \cup P_{2}$. Then $P$ has length $k$ and $P, \rho(P), \rho^{2}(P), \ldots, \rho^{n / 2-1}(P)$ is a $k$-isofactorization of $X$.

This leaves us with the case that $k$ divides $|S|$ but does not divide $|S| / 2$. This implies that $|S|=(2 m+1) k$ for some $m \geqq 1$. If $k$ is odd, then $n$ is even and $n / 2 \in S$. Suppose $S=\left\{ \pm \ell_{1}, \pm \ell_{2}, \ldots \pm \ell_{t}, n / 2\right\}$, where $1 \leqq \ell_{i}<$ $n / 2,1 \leqq i \leqq t$. Make a zig-zag path $P_{1}$ starting at $u_{0}$ and using lengths $\ell_{1}, \ell_{2}, \ldots, \ell_{k}$. Then make a zig-zag path $P_{2}$ starting at $u_{0}$ and using the lengths $\ell_{k+1}, \ell_{k+2}, \ldots, \ell_{2 k}$. Continue with zig-zag paths until $m$ paths $P_{1}, P_{2}, \ldots, P_{m}$ have been constructed that use the first $m k$ lengths of $S$. For each $P_{i}, 1 \leqq$ $i \leqq m$, the paths $P_{i}, \rho\left(P_{i}\right), \rho^{2}\left(P_{i}\right), \ldots, \rho^{n-1}\left(P_{i}\right)$ use all of the edges with lengths $\ell_{1}, \ell_{2}, \ldots, \ell_{m k}$.

The $k$ elements of $S$ not used so far are $\pm \ell_{m k+1}, \ldots, \pm \ell_{t}$ together with $n / 2$. Start zig-zag paths of length $(k-1) / 2$ at both $u_{0}$ and $u_{n / 2}$ and add the edge from $u_{0}$ to $u_{n / 2}$ to produce a path $P$ of length $k$. The $k$-isofactorization is given by the paths $P, \rho(P), \ldots, \rho^{n / 2-1}(P)$.

If $k$ is even, then $n$ must be even because $k$ does not divide $|S| / 2$, and $n / 2 \notin S$ because $|S|$ is even. We know that $k / 2$ divides $|S| / 2$ which means that we can partition the $|S| / 2$ lengths of $S$ into parts $\left\{\ell_{i_{1}}, \ell_{i_{2}}, \ldots, \ell_{i_{k / 2}}\right\}$ of cardinality $k / 2$. For each part, place a zig-zag path $Q_{1}$ of length $k / 2$ using the lengths in the part at $u_{0}$. Let $Q_{2}=\rho^{n / 2}\left(Q_{1}\right)$. The subgraph $Q_{1} \cup Q_{2}$ is rotated through $n / 2$ positions to use all edges of the lengths in the part used to construct $Q_{1}$. This completes the proof.

The glaring omission of Theorem 2.8 is $k=n$. This will be discussed later.

\section{$3 \quad$ Earlier Results}

We now indicate quickly how to obtain two earlier results about isofactorizations. The first result is given in [11].

3.1 Theorem. If $X=\operatorname{circ}(n ; S)$ is a circulant graph with valency 4 and $k$ is any divisor of $|E(X)|$, then $X$ has a k-isofactorization. 
Proof. It is clear that it suffices to prove the result for connected circulant graphs. Hence, we assume $X$ is connected. The number of edges in $X$ is $2 n$. If $k=2 n, X$ itself is the isofactor. If $k=n$, then the main theorem of [3] implies that $X$ has a decomposition into two Hamilton cycles. This takes care of $k=2 n$ and $k=n$.

If $k$ is a proper divisor of $n$ or $k=2$, then the result follows from Theorem 2.8. This leaves us with divisors of the form $2 d$, where $d$ is a proper divisor of $n$. In this case, we use Theorem 2.7 with the proper divisor $n / d$.

The second result appears in [10].

3.2 Theorem. If $X=\operatorname{circ}(n ; S)$ is a circulant graph with valency $p$, where $p$ is a prime, and $k$ is any divisor of $|E(X)|$, then $X$ has a $k$-isofactorization.

PROOF. It is clear that it suffices to prove the result for connected circulant graphs. Hence, we assume $X$ is connected. If $p=2$, then $X$ is a Hamilton cycle and it is easy to see the conclusion is valid in this case. Hence, we assume that $p$ is an odd prime for the rest of the proof. The number of edges in $X$ is $n p / 2$ which implies that $n$ is even. Since the valency $p$ is odd, the length $n / 2$ must belong to $S$.

If $k$ is a divisor of $n / 2$, then it is a proper divisor of $n$ and the result follows from Theorem 2.8. If $k=n p / 2$, then the entire graph is the isofactor. The other divisors have the form $d p$, where $d$ is a proper divisor of $n / 2$. Use Theorem 2.7 with the proper divisor $n / 2 d$ to settle the remaining case.

\section{Isofactors with few edges}

We now consider isofactors whose size is smaller than the order of the circulant graph under discussion. If $X=\operatorname{circ}(n ; S)$ and $k<n$ is a divisor of $n|S| / 2$, the results of Section 2 take care of the cases when either $k$ divides $n$ or $k$ divides $|S| / 2$. This leaves us with the situation that neither of these divisibility conditions hold. The next theorem is a partial result whose proof indicates one approach when the isofactors have small size.

4.1 Theorem. Let $X=\operatorname{circ}(n ; S)$ be a circulant graph of order $n$, and let $k$ satisfy $k<n, k$ divides $n|S| / 2$, and $k$ does not divide either $n$ or $|S| / 2$. Let $\bar{n}=\operatorname{gcd}(n, k), \bar{k}=k / \bar{n}$, and $t \bar{k}=|S| / 2$. If either $t=1$, or $t \geqq \bar{n} / 2$ and $S$ has no elements divisible by $\bar{n}$, then $X$ has a $k$-isofactorization. Moreover, when $t \neq 1$, we obtain isofactors that are vertex-disjoint unions of $\bar{n}$ paths of length $\bar{k}$.

Proof. We use the notation in the statement of the theorem. Note that $\bar{n}<n$ because $k<n$. On the other hand, $\bar{n}=1$ implies that $k$ divides $|S| / 2$ which is contrary to hypothesis. Thus, $1<\bar{n}<n$.

The definition of $\bar{k}$ implies that we want isofactors with $\bar{n} \bar{k}$ edges. When $t \neq 1$, we achieve this by producing isofactors that are vertex-disjoint unions of 
$\bar{n}$ paths of length $\bar{k}$. Before handling the general case, we dispose of the case that $t=1$.

We have $\bar{k}=|S| / 2$ when $t=1$, so that $|S| / 2$ is an integer. This implies that $|S|$ is even. In particular, the element $n / 2$ does not belong to $S$ when $n$ is even. Because the number of isofactors we want is a divisor of $n$, we invoke Theorem 2.7.

Suppose that $t>1$. It is convenient to arrange the vertices of $X$ as an array with $n / \bar{n}$ rows and $\bar{n}$ columns. The vertices in the first column, starting with the top row and working down the column, are $u_{0}, u_{1}, u_{2}, \ldots, u_{\bar{n}-1}$. The vertices across the rows are the orbits of $\rho^{n / n}$ acting on $V(X)$ listed cyclically from the element of the row in the first column. Whenever we can find a path $P$ of length $\bar{k}$ that uses at most one vertex from each row of the array, then the paths $P, \rho^{n / n}(P), \ldots, \rho^{n-n / \bar{n}}(P)$ are vertex-disjoint because $P$ uses at most one vertex of each orbit of $\rho^{n / \bar{n}}$.

When we have a collection of distinct lengths, using the lengths in a zig-zag fashion guarantees that we obtain a path. If we think of the rows of the array as ordered cyclically (that is, place the rows around a cylinder), then we can talk about the length between rows too. Unfortunately, distinct lengths in $X$ may have the same length between rows. Thus, care must be used when trying to construct paths of length $\bar{k}$ in the array that use at most one vertex from each row.

We partition the $|S| / 2$ lengths of $X$ into $t$ subsets $L_{1}, L_{2}, \ldots, L_{t}$. Note that at most $\bar{n}$ lengths of $X$ have the same length between rows of the array. Hence, when we partition the lengths of $X$, we can ensure that at most two lengths of any $L_{i}$ have the same length between rows of the array because $2 t \geqq \bar{n}$.

We now prove a general claim about multisets of lengths and apply it to complete the proof of the theorem. To that end, consider a multiset of $r$ lengths with no length appearing more than twice, and where $\ell$ is the longest length that occurs. Then we claim we can find a path of length $r$ in $K_{N}$ using each of the lengths as long as $N \geqq 2 \ell$ when the length $\ell$ appears once, or $N \geqq 2 \ell+1$ when the length $\ell$ appears twice.

Let $d_{1}<d_{2}<\cdots<d_{s}$ be all of the distinct lengths of the multiset arranged in ascending order. Note that $d_{s}=\ell$. Let $d_{1}^{\prime}<d_{2}^{\prime}<\cdots<d_{s^{\prime}}^{\prime}$ be the duplicated lengths arranged in ascending order. Starting at $v_{0}$ construct the zig-zag $Q$ path using the lengths $d_{1}, d_{2}, \ldots, d_{s}=\ell$. All the vertices of $Q$ lie inside a segment of vertices for which the edge of length $\ell$ is a chord. Starting from the end vertex of the edge of length $d_{s}$ that also is an end vertex of $Q$, construct a zig-zag path using edges of lengths $d_{s^{\prime}}^{\prime}$ down to $d_{1}^{\prime}$ in decreasing order. There is enough room to complete the path because of the conditions on $N$ in relation to how many times $\ell$ occurs as a length. The resulting path is the path we are looking for and shows that the claim is true.

Using the claim, the assumption there is no length divisible by $\bar{n}$, and the assumption that no length between the rows appears more than twice in any $L_{i}$, we construct a path $P_{i}$ of length $\bar{k}$ using at most one vertex of any row and using the lengths of $L_{i}$. Then $P_{i}, \rho^{n / \bar{n}}\left(P_{i}\right), \ldots, \rho^{n-n / \bar{n}}\left(P_{i}\right)$ is a vertexdisjoint union of $\bar{n}$ paths of length $\bar{k}$. This is then the isofactor $X_{i}$. The 
subgraphs $X_{i}, \rho^{\bar{n}}\left(X_{i}\right), \ldots, \rho^{n-\bar{n}}\left(X_{i}\right)$ use all of the edges of $X$ of the lengths in $L_{i}$. Therefore, we have found a $k$-isofactorization of $X$ into subgraphs that are vertex-disjoint unions of $\bar{n}$ paths of length $\bar{k}$.

An intriguing case is that of isofactors with $|V(X)|$ edges. It has been conjectured [2] that every connected circulant graph of even valency has a Hamilton decomposition. A positive answer to this conjecture, of course, implies that we can always find a $|V(X)|$-isofactorization for any circulant graph of even valency. (If a circulant graph $X$ has odd valency, then $|V(X)|$ does not divide $|E(X)|$ anyway.) The following theorem was proved in [3].

4.2 Theorem. A connected circulant graph of valency 4 has a decomposition into two Hamilton cycles.

The connection set $S$ for a circulant graph $\operatorname{circ}(n ; S)$ is said to be minimal if $S \backslash\{ \pm s\}$ generates a proper subgroup of $\mathbb{Z}_{n}$ for every $s \in S$. J. Liu proved the following result in [8].

4.3 Theorem. Let $X=\operatorname{circ}(n ; S)$ be a connected circulant graph of odd order. If $S$ is minimal, then $X$ has a Hamilton decomposition.

4.4 Corollary. Let $X=\operatorname{circ}(n ; S)$ be a connected circulant graph. If $|S|$ is even and its elements can be partitioned into parts of the form $\left\{ \pm s_{1}, \pm s_{2}\right\}$ so that $\operatorname{gcd}\left(n, s_{1}, s_{2}\right)=1$ for each pair, then $X$ has a Hamilton decomposition. The same conclusion holds when $|S|$ is odd, if $S$ can be partioned into parts as before together with a part of the form $\{ \pm s\}$, where $\operatorname{gcd}(s, n)=1$.

Proof. It is well known that the circulant graph $\operatorname{circ}\left(n ;\left\{ \pm s_{1}, \pm s_{2}\right\}\right)$ is connected if and only if $\operatorname{gcd}\left(n, s_{1}, s_{2}\right)=1$. We apply Theorem 4.2 to decompose this circulant into two Hamilton cycles. Doing this for each of the parts produces a Hamilton decomposition of $X$ when $|S|$ is even. When $|S|$ is odd, we have a single Hamilton cycle coming from the element $s$.

The preceding results cover many cases when we are seeking isofactors with $|V(X)|$ edges, unfortunately, it is easy to find examples that are not covered by the preceding results. The circulant graph $\operatorname{circ}(15,\{ \pm 3, \pm 5, \pm 6\})$ is one such example. This invites us to seek other methods for producing a $|V(X)|-$ isofactorization.

4.5 Corollary. Let $X=\operatorname{circ}(n ; S)$ be a circulant graph with $|S|$ even. If $|S| / 2$ divides $n$, then $X$ has an $n$-isofactorization.

Proof. This follows directly from Theorem 2.7 by letting $d=|S| / 2$.

We turn our attention to the valency 6 case. There is some new work by Dean $[4,5]$ that almost settles this case. We summarize his results in the next theorem. 
4.6 Theorem. Let $X=\operatorname{circ}(n ; S)$ be a connected circulant graph of valency 6 . If $n$ is odd, or $n$ is even and some element $s \in S$ satisfies $\operatorname{gcd}(n, s)=1$, then $X$ has a Hamilton decomposition.

Circulant graphs of even order with connection set $S=\left\{ \pm s_{1}, \pm s_{2}, \pm s_{3}\right\}$, where $\operatorname{gcd}\left(n, s_{i}\right)>1$, for $i=1,2,3$, and $\operatorname{gcd}\left(n, s_{1}, s_{2}, s_{3}\right)=1$, are the only cases not covered by the preceding theorem. When these conditions hold, Theorem 2.7 gives an $n$-isofactorization when $n$ is a multiple of 3 by using $d=3$ in the theorem. As a matter of fact, we also can do the remaining cases for even $n$, but the proof is lengthy and is omitted here.

\section{$5 \quad$ Small order circulant graphs}

We conclude the paper by examining circulant graphs of small order. It is not difficult to see that it suffices to consider only connected circulant graphs. The case of valency 1 is covered by Lemma 2.2. The cases of valencies $2,3,4,5$, and 7 are covered by Theorems 3.1 and 3.2 .

For valency 6 , we have $|E(X)|=3 n$, where $n$ is the order of the circulant graph. If 3 divides $k$, then Theorem 2.7 establishes the existence of a $k$-isofactorization of $X$. If 3 does not divide $k$, then Theorem 4.1 covers all situations except $k=n$. For the latter, Theorem 4.6 takes care of $n$ odd and any cases for $n$ even with at least one $s \in S$ satisfying $\operatorname{gcd}(n, s)=1$. There are examples of circulant graphs of orders 12 and 18 that are connected, have valency 6 , and have no elements of the connection set relatively prime to $n$. However, the number of required isofactors is three which divides $n$, so they are covered by Theorem 2.7.

Thus, for valency 6 there are no counterexamples of order smaller than 20 . We move to valency 8 . Since [7] settles the case for complete graphs, the smallest examples not covered have order at least 10 . If $X$ has order 10 and valency 8 , it has 40 edges. The divisors 2,4 and 5 are covered by Theorem 2.8. The divisors 8 and 20 are covered by Theorem 2.7. The divisor 10 is covered by Theorem 4.4. The other divisors are trivial.

There is one circulant graph, to within isomorphism, of order 11 and valency 8. The methods of this paper fail to provide a 22-isofactorization for this circulant graph. The next smallest examples are the circulant graphs of order 13 and valency 8 with $k=26$. The smallest even order circulant graphs whose isofactorizations are not covered by this paper have order 14 and valency 12 with $k=21,28$. We have found appropriate isofactorizations using ad-hoc methods.

ACKnowledgement. The authors wish to thank Darryn Bryant for referring us to reference [6]. The last author wishes to thank the Department of Mathematics and Statistics at the University of Regina for its hospitality while this research was being completed. 


\section{References}

[1] B. Alspach, Research Problem 19, Discrete Math. 40 (1982), 321-322.

[2] B. Alspach, Research Problem 59, Discrete Math. 59 (1984), 115.

[3] J.-C. Bermond, O. Favaron and M. Maheo, Hamiltonian decomposition of Cayley graphs of degree four, J. Combin. Theory Ser. B 46 (1989), $142-153$.

[4] M. Dean, Hamilton cycle decomposition of 6-regular circulants of odd order, preprint.

[5] M. Dean, On hamilton cycle decompositions of 6-regular circulants, preprint.

[6] M. Ellingham and N. Wormald, Isomorphic factorization of regular graphs and 3-regular multigraphs, J. London Math. Soc.(2) 37 (1988), 14-24.

[7] F. Harary, R. W. Robinson and N. C. Wormald, Isomorphic factorisations I: Complete graphs, Trans. Amer. Math. Soc. 242 (1978), 243-260.

[8] J. Liu, Hamiltonian decompositions of Cayley graphs on abelian groups of odd order, J. Combin. Theory Ser. B 66 (1996), 75-86.

[9] R. Stong, On 1-factorizability of Cayley graphs, J. Combin. Theory Ser. B 39 (1985), 298-307.

[10] J. F. Wang and Y. S. Zhou, Isomorphic factorization of circulant graphs with prime degree, Chinese Quart. J. Math. 3 (1988), 66-70.

[11] C. G. Yan and D. L. Chen, Isomorphic factorization of circulant graphs with degree 4, (Chinese) Shandong Kuangye Xueyuan Xuebao 14 (1995) 196-200. 